\title{
On the effects of build orientation, strain rate sensitivity and sample thickness on the mechanical behavior of 316I Stainless Steel manufactured by Selective Laser Melting
}

\author{
Hugo Carassus ${ }^{1,2}$ *, Hervé Morvan ${ }^{1}$, Gregory Haugou ${ }^{1}$, Jean-Dominique Guerin ${ }^{1}$, Tarik \\ Sadat $^{1}$, Sandra Guerard ${ }^{2}$, and Eric Markiewicz ${ }^{1}$ \\ ${ }^{1}$ Univ. Polytechnique Hauts-de-France, CNRS, LAMIH UMR 8201, 59313 Valenciennes, France \\ ${ }^{2}$ Arts et Métiers ParisTech, CNRS, I2M UMR 5295, 33405 Talence, France
}

\begin{abstract}
The Additive Layer Manufacturing (ALM) for metallic materials has grown in the past few years. However, this process influences the mechanical properties of the constitutive material and consequently those of the finished product. The influence of the thickness and the building direction of 316L Stainless Steel (SS) specimens produced by Selective Laser Melting (SLM) on the quasi-static mechanical behavior has already been reported. Considering the strain rate effect, it has been only studied for tensile properties of vertical specimens up to $10^{2} \mathrm{~s}^{-1}$. The aim of this work is to study the influence of the thickness and the building orientation at higher strain rates up to $10^{1} \mathrm{~s}^{-1}$ and up to $10^{3} \mathrm{~s}^{-1}$ for vertical specimens. Compared to conventional material, 316L SS SLM achieves equal and even better mechanical properties due to a refinement of the microstructure. Anisotropy is observed at the macroscopic level, which is explained by the microstructure with different shapes, orientation and size of grains. A minimum thickness of $0.75 \mathrm{~mm}$ is recommended to recover the mechanical properties of the conventional $316 \mathrm{~L} \mathrm{SS}$. A positive strain rate sensitivity is observed in every case. The material anisotropy and the thickness variation do not affect the strain rate sensitivity.
\end{abstract}

\section{Introduction}

Metallic additive manufacturing processes like Selective Laser Melting (SLM) give access to the microscopic scale while keeping a high level of complexity in geometry. It offers the possibility to design lighter and stiffer structures, with good energy absorption capacities, such as lattice or Triply Periodic Minimal Surfaces (TPMS).

SLM is a power bed fusion process with a layer-by-layer build-up method allowing production of monolithic design whereas other conventional process would not be adapted. The specific microstructure produced by this process due to high cooling rates has a strong influence on the mechanical behavior and need to be understood for further industrial

\footnotetext{
*Corresponding author: hugo.carassus@uphf.fr
} 
applications [1]. Among the different alloys used in SLM, the 316L Stainless Steel (SS) is considered in this study for his high strength-ductility combination [2].

Several works have shown that constitutive materials manufactured by SLM are not fully isotropic. Tensile test specimens built vertically (along the building direction) have higher flow stress but lower failure strain than horizontally built specimens. Although the trend is accepted for these two building orientations, is it unclear for intermediate angles [3].

The influence of the specimen thickness on the mechanical response of plate samples has been investigated by few studies [4-6]. It has been shown that the mechanical strength increases with the thickness. Roach et al [5] noticed an increase of $50 \%$ of the effective strength and stress for thicknesses from $0.4 \mathrm{~mm}$ to $6.25 \mathrm{~mm}$. The strain rate sensitivity of $316 \mathrm{~L}$ SS SLM studied on plate specimens built vertically has been measured up to strain rate of $10^{2} \mathrm{~s}^{-1}$ [7-9]. Li et al [9] observed an increase of the Yield Stress (YS) of $26 \%$ between $0.001 \mathrm{~s}^{-1}$ and $193 \mathrm{~s}^{-1}$ strain rates.

To the best of our knowledge, all published works on the thickness and building orientation of 316L SS manufactured by SLM were conducted under quasi-static tensile loading. The present study will aim to characterize $316 \mathrm{~L}$ SS SLM specimens with different building orientations and thicknesses at multiple strain rates. Three thicknesses of $0.5-0.75-1 \mathrm{~mm}$ on vertical specimens and three building orientations of $0^{\circ}$ (horizontal) - $45^{\circ}$ (diagonal) $90^{\circ}$ (vertical) with $1 \mathrm{~mm}$ thickness will be considered and samples will be tested at three strain rates: $0.00057 \mathrm{~s}^{-1}, 0.067 \mathrm{~s}^{-1} \mathrm{~s}^{-1}$ and $8 \mathrm{~s}^{-1}$. To quantify the strain rate sensitivity, vertical specimens will be tested with the three previous strain rates and two higher ones: $300 \mathrm{~s}^{-1}$ and $1000 \mathrm{~s}^{-1}$. Additionally, a microstructure analysis will be conducted to explain the macroscopic results. The findings can be used for further development of design rules and prediction of mechanical properties of SLM parts containing design features with lower wall thicknesses and different building orientations.

\section{Material and Methods}

A SLM 280 machine from SLM-Solution was used to build specimens with a $316 \mathrm{~L}$ SS powder with a spherical shape and a particle size ranging from $15 \mu \mathrm{m}$ to $45 \mu \mathrm{m}$. The selected scan strategy was single layer meandering and, to produce near full dense samples, the main process parameters were: laser power $200 \mathrm{~W}$, scanning speed $800 \mathrm{~mm} . \mathrm{s}^{-1}$, hatch spacing $120 \mu \mathrm{m}$, layer thickness $30 \mu \mathrm{m}$. After fabrication, the specimens were sandblasted, thermally treated for two hours at $1095^{\circ} \mathrm{C}$ and then water quenched. Samples with a thickness of $0.5 \mathrm{~mm}$ and $0.75 \mathrm{~mm}$ were not post treated to avoid warping. The specimens were named after their building orientation and their thickness. Thus Bd90_t075 was built vertically, parallel to the building direction, with a thickness of $0.75 \mathrm{~mm}$.

The specimens were sectioned longitudinally along the tensile direction and coated with black bakelite hot mounting resin with carbon filler from Struers. Then samples were polished following Struers recommendation for metallographic preparation with sand papers from $220 \mu \mathrm{m}$ to $1 \mu \mathrm{m}$ to achieve a mirror surface. To reveal melt pools and grain boundaries, samples were etched with Villela solution for 30 seconds, then observed with an optical microscope Nikon Epiphot 200 and a Scanning Electron Microscope (SEM) JOEL JSM$7100 \mathrm{~F}$ Field Emission. The study of the grain shape, orientation and size was performed using SEM and OXFORD Electron BackScatter Diffraction (EBSD) detector. For EBSD analysis, after being polished to obtain a mirror surface, samples were etched with Struers OP-S Chem solution for two minutes. EBSD data were analyzed using the AZTec (UK) software from OXFORD instruments

Quasi-static tensile tests were done on a Instron Sintech, an electromechanical testing machine, with a $30 \mathrm{kN}$ load cell at a loading rate of $1 \mathrm{~mm} / \mathrm{min}\left(0.00057 \mathrm{~s}^{-1}\right)$. Instron VHS, a servohydrolic testing machine, with a $30 \mathrm{kN}$ load cell was used to increase the loading rate to 
$2 \mathrm{~mm} . \mathrm{s}^{-1}\left(0.067 \mathrm{~s}^{-1}\right)$ and $240 \mathrm{~mm} . \mathrm{s}^{-1}\left(8 \mathrm{~s}^{-1}\right)$. Split Hopkinson Tension Bars (SHTB) were used to reach the highest two loading rates of $3 \mathrm{~m} . \mathrm{s}^{-1}\left(300 \mathrm{~s}^{-1}\right)$ and $10 \mathrm{~m} . \mathrm{s}^{-1}\left(1000 \mathrm{~s}^{-1}\right)$. A digital camera Manta Allied Vision was used for quasi-static tests and a Fastcam Photron APX RS was used for higher strain rates. Displacement and deformation were calculated by Digital Image Correlation with an intern MATLAB script.

\section{Results and discussion}

\subsection{Tensile test results}

Mean stress-strain curves and the associated mechanical parameters are presented in Fig. 1. Horizontal specimens have higher Yield Stress (YS) and Ultimate Tensile Stress (UTS) but lower failure strain $\left(\varepsilon_{\mathrm{f}}\right)$, at the opposite of vertical samples. Diagonal specimens are in between the two previous ones. Bd90_t05 has lower YS and UTS of all configurations. As the strain rate increases, YS and UTS increase but not $\varepsilon_{\mathrm{f}}$. Mechanical parameters in this study are consistent with the large scatter in the literature, which reflects the variety of possible process parameters combinations which influences the mechanical performances $[1,9,10]$. Compared to conventional 316L SS and the associated norm ASTM A240/A240M [11] (YS $\geq 170 \mathrm{MPa}, \mathrm{UTS} \geq 485 \mathrm{MPa}$ and $\varepsilon_{\mathrm{f}} \geq 40 \%$ ), higher YS and UTS are achieved by SLM samples (except for $0.5 \mathrm{~mm}$ thick samples) and similar $\varepsilon_{\mathrm{f}}$ (except for Bd0_t1).

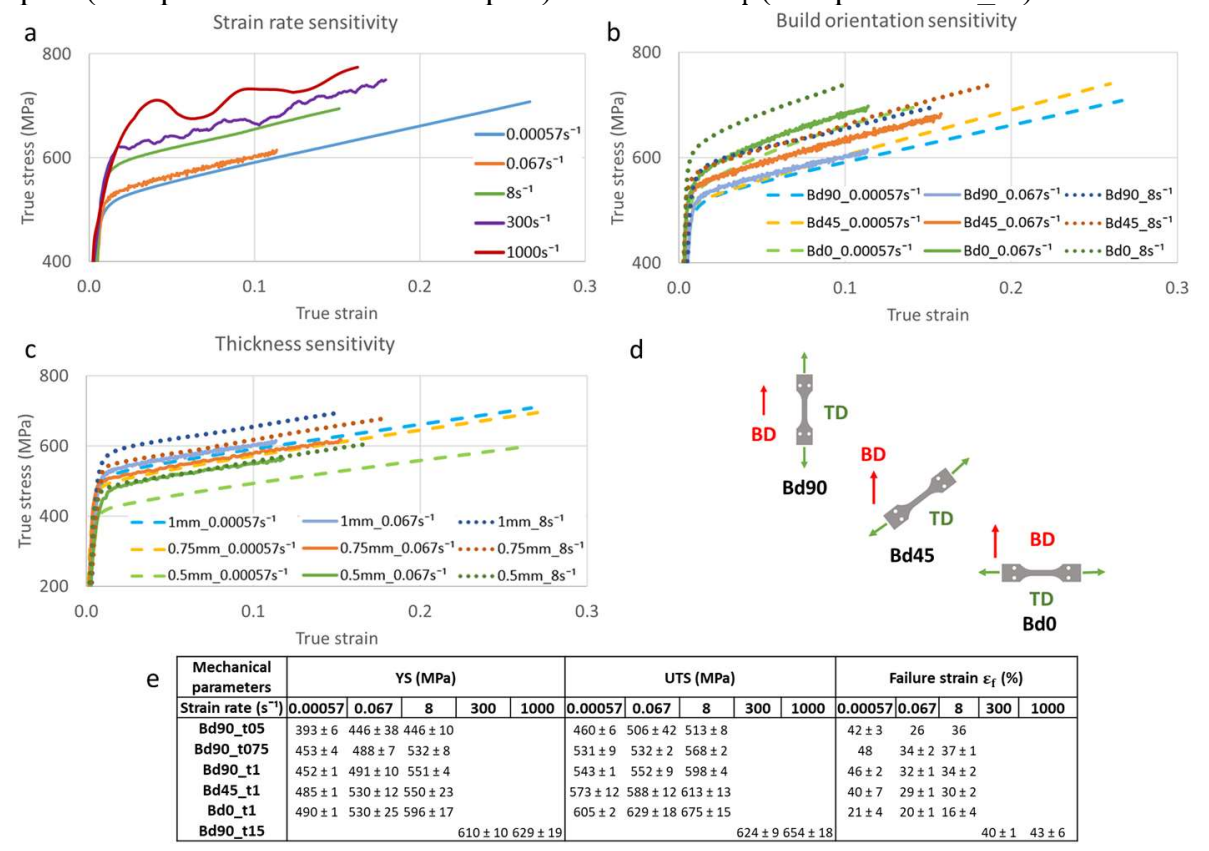

Fig. 1. True stress-strain curves of strain rate sensitivity of vertical samples (a), building orientation sensitivity of $1 \mathrm{~mm}$ thick samples (b), thickness sensitivity of vertical samples (c) and a representation of the three building orientations along the Tensile Direction (TD) and the Building Direction (BD). Conventional mechanical parameters of all configurations (e).

\subsection{Microstructure}

Optical micrographs of 316L SS specimens in Fig. 2, along the building direction, show stacking of layers with multiple overlapping melt pools that have an elliptical shape. At the 
center of the samples, melt pools are approximately $100 \mu \mathrm{m}$ deep, which typical of SLM laser melt tracks [12]. These images also present the defects than can occur during the process. These defects are mainly unmelted particles of powder which were pulled out from the sample during the polishing. The spherical shape and the similar size, $15 \mu \mathrm{m}$ to $45 \mu \mathrm{m}$, confirm this observation. Bd90_t 05 is the only configuration that has a relative density below $99 \%$, which is the minimum recommended to consider that defects are not detrimental to mechanical properties [7]. This can be explained by the higher density of porosity compared to other configurations.
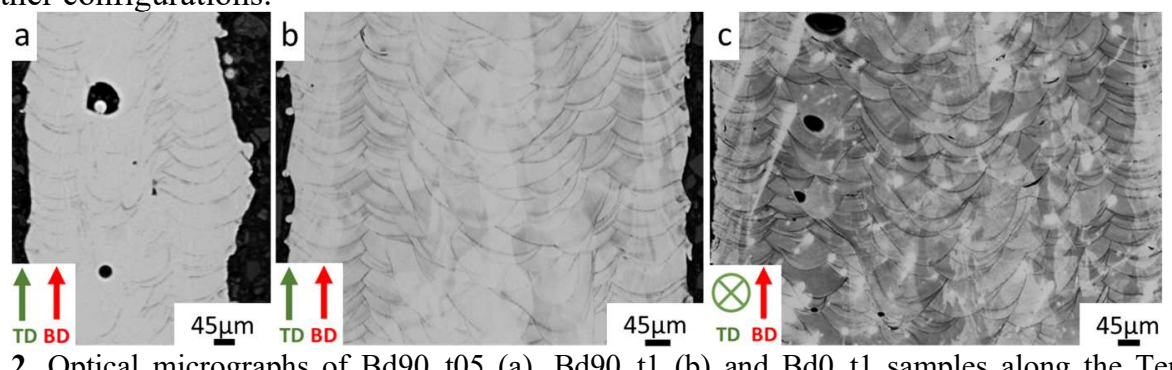

Fig. 2. Optical micrographs of Bd90_t05 (a), Bd90_t1 (b) and Bd0_t1 samples along the Tensile Direction (TD) with respect to the Building Direction (BD).

The microstructure of a melt pool (Fig. 3a) shows the fine cellular structure, with a grain size considered to be in between $0.1-2 \mu \mathrm{m}$. This is caused by the high-cooling rate, in the order of $10^{4}-10^{6} \mathrm{~K}^{-\mathrm{s}^{-1}}$, which generates non-equilibrium solidification conditions [12]. During the solidification process, the dendritic structure is oriented along the maximal local thermal gradient, which can deviate from the macroscopic one, resulting in different orientations inside a melt pool. The subgrain close to the melt pools boundaries (Fig. 3b) are coarser due to the multiple melting.

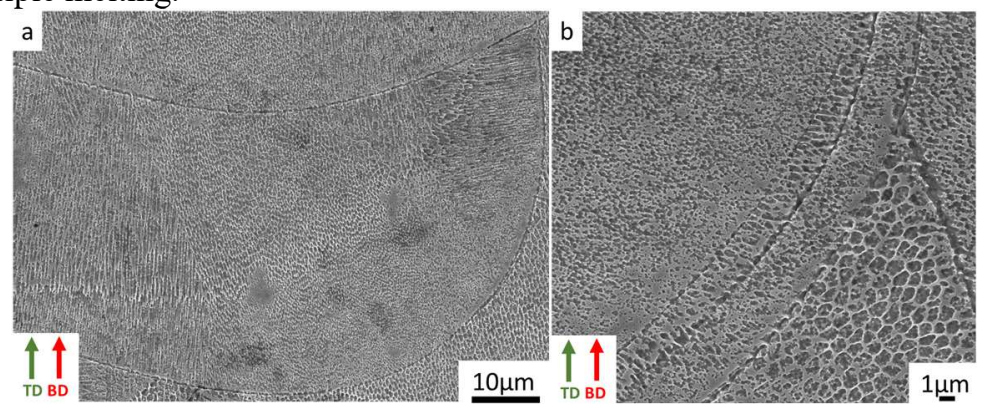

Fig. 3. SEM images of Bd0_t1 at two magnifications showing the subgrain structure inside a meltpool (a) and close to a melt pool boundary (b) along the Tensile Direction (TD) with respect to the Building Direction (BD).

Fig. 4 shows representative EBSD inverse pole figure of the three buiding directions specimens along the tensile direction. The average grain size computed from the EBSD images are $14 \mu \mathrm{m}$ for $\mathrm{Bd} 0 \_\mathrm{t} 1,28 \mu \mathrm{m}$ for $\mathrm{Bd} 90 \_\mathrm{t} 1$ and $17 \mu \mathrm{m}$ for $\mathrm{Bd} 45 \mathrm{t} 1$. Those values are typical of the SLM process and smaller than the conventional $316 \mathrm{~L}$ SS with a grain size in the range of $30-80 \mu \mathrm{m}$ and a more homogeneous grain structure [10]. There is a clear distinction between horizontal and vertical microstructure. The fist one has narrow and elongated grains perpendicular to the tensile direction, whereas the second one has deeper grains oriented toward the tensile direction with a grain size twice as high. Diagonal specimens, as to them, is a combination of the two previous ones with grains oriented at $45^{\circ}$ with respect to the tensile direction. In the three cases, grains cross multiple melt pools, which is representative of epitaxial growth. This is caused by remelting of layers that dictates the same crystallographic orientation [12]. No texturation is discernible on the three EBSD 
images and for other configurations. Suryawanshi et al [1] revealed that although several works report a texturation, it was not the case in their results, as it is the case in our study.

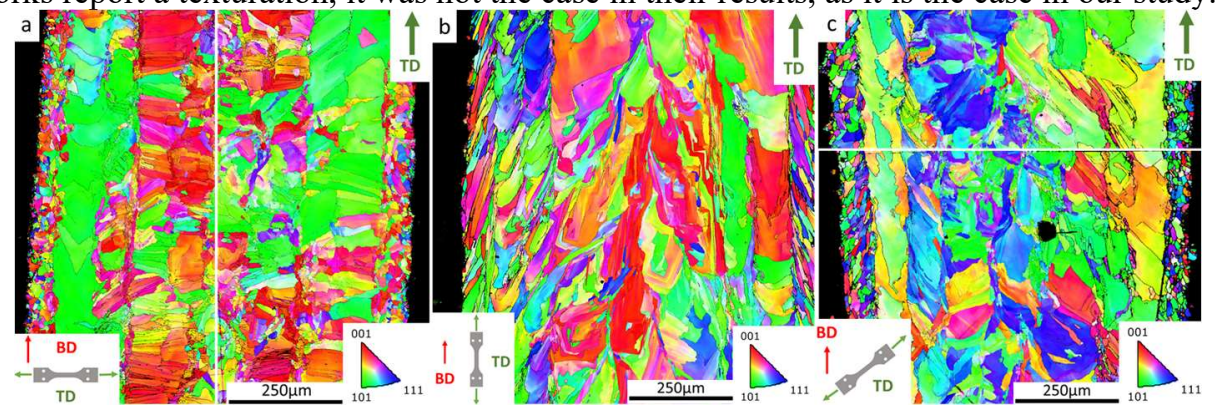

Fig. 4. EBSD images of Bd0_t1 (a), Bd90_t1 (b) and Bd45 t1 (c) along the Tensile Direction (TD).

\subsection{Discussion}

SLM studied in this work produce parts with a unique microstructure which impacts the macroscopic behavior. In the case of tensile tests, 316L SS SLM specimens tend to have higher YS than conventional material, and equivalent UTS and failure strain. Factors such as cellular dendritic structure (subgrain) and smaller grain size $(20-30 \mu \mathrm{m}$ for SLM against 30$100 \mu \mathrm{m}$ for conventional) help understanding these enhancements [1].

All samples exhibit a positive strain rate sensitivity. The YS and UTS increase by $19 \pm 3 \%$ and $11 \pm 2 \%$ between $0.00057 \mathrm{~s}^{-1}$ and $8 \mathrm{~s}^{-1}$ in average for all configurations. For vertical samples, it increases by $42 \%$ for YS and $22 \%$ for UTS between $0.00057 \mathrm{~s}^{-1}$ and $1000 \mathrm{~s}^{-1}$. The conventional material is less impacted by the strain rate effect [8]. The authors explained that the higher strain rate sensitivity of the SLM material is caused by the grain refinement. Differences observed with the three building orientations represent $11 \%$ for YS and $14 \%$ for UTS at each strain rate. Li et al [9] noticed a variation of $\pm 10 \%$ for the YS at $0.001 \mathrm{~s}^{-1}$ strain rate between the same build orientations and concluded to an anisotropic behavior. Conventional 316L SS studied by Ghosh and Gurao et al [13] had differences between the three building orientations of $3 \%$ for the YS and $2 \%$ for the UTS. These smaller variations indicate a lower anisotropy behavior compared to SLM parts. The grain shape, orientation and size explain the anisotropy observed at the macroscopic scale. Under uniaxial tensile test, in the case of Bd90 specimens, dislocations have an inferior amount of grain boundaries to cross than Bd0 specimens. It thus explained the higher YS and UTS. The lower failure strain of horizontal specimens is possibly linked to the interlayers perpendicular to the tensile direction which are ideal crack initiation sites. Charmi et al [6] concluded similarly and also suggested that the different deformation mechanisms, as twinning or dislocation glide, are responsible for directional dependency of the material behavior.

Considering the influence of the thickness, a lower flow stress is observed for $0.5 \mathrm{~mm}$ thickness compared to $0.75 \mathrm{~mm}$ and $1 \mathrm{~mm}$ thicknesses, whatever the strain rate (Fig. 1c). With thicknesses from $0.4 \mathrm{~mm}$ to $6.25 \mathrm{~mm}$, Roach et al [5] showed that the YS and UTS decreased when reducing sample cross-section. The higher standard deviation for YS and UTS is also noticed for Bd90_t05, which is likely to be caused by the higher density of defects than other samples. The strain rate sensitivity is similar for $0.75 \mathrm{~mm}$ and $1 \mathrm{~mm}$ thick specimens whereas it is less obvious for $0.5 \mathrm{~mm}$ thickness, again possibly due to the defects. Leicht et al [4] explained that reduction of the mechanical properties at lower thicknesses are caused by different temperature history during manufacturing resulting in distinct microstructures. As there is a huge thermal conductivity difference between the metal powder and the deposited bulk material the higher the thickness, the higher amount of heat can be dissipated [2]. It thus leads to a more homogenous microstructure. 


\section{Conclusion}

In this work, the influence of strain rate, building orientation and thickness on the mechanical behavior of 316L SS SLM specimens have been investigated. Based on the microstructure analysis, uniaxial tensile tests results and previous published works, the main findings are summarized as follows:

- Specimens produces by SLM achieves better YS, similar UTS and equal failure strain compared to conventional material (except for Bd90_t05 specimens). The explanations are found in the microstructure with the presence of subgrain structure and smaller grain size.

- The building orientation results in a variation of $10 \%$ in the YS and $14 \%$ in the UTS at each strain rate, the higher values being achieved by horizontal specimens. However, the failure strain is nearly divided by two for the latter compared to the two other build orientations. The microstructure reveals important differences regarding orientation, size and shape of grains causing this anisotropy.

- A minimum thickness of $0.75 \mathrm{~mm}$ is recommended to achieve as good and even better mechanical properties than conventional material, which is not the case for $0.5 \mathrm{~mm}$ thickness. The microstructure of the thin part has a higher porosity and a reduced cohesion between grains and layers.

This work is supported by Institut Carnot ARTS and Haut-de-France region.

\section{References}

1. J. Suryawanshi, K.G. Prashanth, U. Ramamurty. Mater Sci Eng A, 696, 113-21 (2017)

2. X. Wang, J.A. Muñiz-Lerma, O. Sánchez-Mata, M. Attarian Shandiz, M. Brochu, Mater Sci Eng A, 736, 27-40 (2018)

3. L. Hitzler, J. Hirsch, B. Heine, M. Merkel, W. Hall, A. Öchsner, Materials (Basel), 10 (2017)

4. A. Leicht, C. Pauzon, M. Rashidi, U. Klement, L. Nyborg, E. Hryha, Adv Ind Manuf Eng, 2, 100037 (2021)

5. A.M. Roach, B.C White, A. Garland, B.H. Jared, J.D. Carroll, B.L. Boyce, Addit Manuf, 32, 101090 (2020)

6. A. Charmi, R. Falkenberg, L. Ávila, G. Mohr, K. Sommer, A. Ulbricht, Mater Sci Eng A, 799, 140154 (2021)

7. Z. Li, T. Voisin, J.T. McKeown, J. Ye, T. Braun, C. Kamath, Int J Plast, 120, 395-410 (2019)

8. F. Khodabakhshi, M.H. Farshidianfar, A.P. Gerlich, M. Nosko, V. Trembošová, A. Khajepour, Mater Sci Eng A, 756, 545-61 (2019)

9. X. Li, C.C Roth, T. Tancogne-Dejean, D. Mohr, Int J Impact Eng, 145, 103671 (2020)

10. R. Shrestha, J. Simsiriwong, N. Shamsaei, S.M. Thompson, L. Bian, 27th Annual Solid Frefform Fabrication Symposium Proceedings, 605-16 (2016)

11. ASTM A240/A240M-20a, ASTM International (2020)

12. A. Chniouel, P.F. Giroux, F. Lomello, P. Aubry, É. Vasquez, O. Hercher, Int J Adv Manuf Technol, 111, 3489-503 (2020)

13. A. Ghosh, N.P. Gurao. Mater Des, 109, 186-96 (2016) 